\title{
Biocatalysts from Biosynthetic Pathways: Enabling Stereoselective, Enzymatic Cycloether Formation on a Gram Scale
}

Tim Hollmann," Gesche Berkhan," Lisa Wagner, Kwang Hoon Sung, Simon Kolb, Hendrik Geise, and Frank Hahn*

Cite This: ACS Catal. 2020, 10, 4973-4982

Read Online

\section{ACCESS I}

Wlll Metrics \& More

Article Recommendations

Supporting Information

ABSTRACT: Biosynthetic pathways of natural products contain many enzymes that contribute to the rapid assembly of molecular complexity. Enzymes that form complex structural elements with multiple stereocenters, like chiral saturated oxygen heterocycles $(\mathrm{CSOH})$, are of particular interest for a synthetic application, as their use promises to significantly simplify access to these elements. Here, the biocatalytic characterization of $\mathrm{AmbDH} 3$, an enzyme that catalyzes intramolecular oxa-Michael addition (IMOMA) is reported. This reaction essentially gives access to various types of $\mathrm{CSOH}$ with adjacent stereocenters, but it is not yet part of the repertoire of preparative biocatalysis. An in-depth study on the synthetic utility of $\mathrm{AmbDH} 3$ was performed, which made extensive use of complex

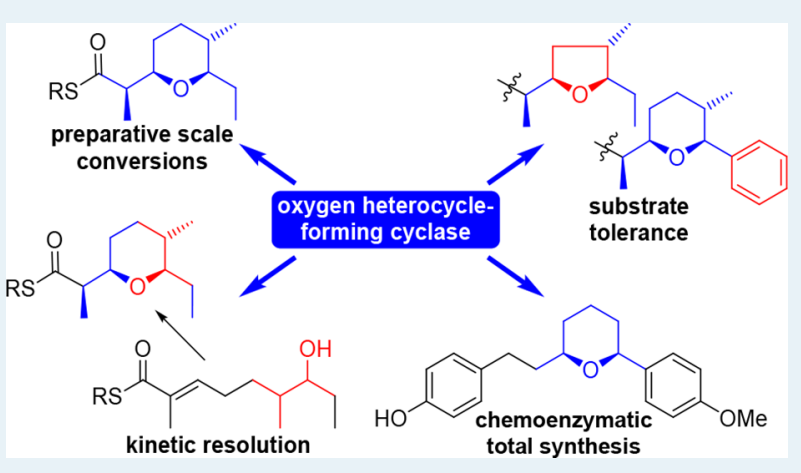
synthetic precursor surrogates. The enzyme exhibited stability and broad substrate tolerance in in vitro experiments, which was in agreement with the results of molecular modeling. Its selectivity profile enabled kinetic resolution of chiral tetrahydropyrans (THPs) under control of up to four stereocenters. A systematic optimization of the reaction conditions enabled gram-scale conversions yielding preparative amounts of chiral THP. The synthetic utility of AmbDH3 was finally demonstrated by its successful application in the key step of a chemoenzymatic total synthesis to the THP-containing phenylheptanoid (-)-centrolobine. These results highlight the synthetic potential of AmbDH3 and related IMOMA cyclases as a biocatalytic alternative that further develops the available chemical-synthetic IMOMA methodology.

KEYWORDS: biocatalyst discovery, cyclases, oxa-Michael addition, heterocycles, tetrahydropyrans, natural product (bio)synthesis, new enzymatic activity

\section{INTRODUCTION}

Natural product biosynthetic pathways are a rich source of enzymes with unprecedented activities. ${ }^{1,2}$ Among them, enzymes that form complex molecular fragments with multiple stereocenters, like terpene cyclases, macrocyclizing thioesterases, or heterocycle-forming cyclases, ${ }^{3-5}$ are particularly attractive for biocatalysis, as their selectivity often significantly exceeds that of analogous chemical-synthetic methodology. Their biocatalytic application promises to enable efficient, stepeconomic access to complex natural products, synthetic drugs, and high-value chemicals. ${ }^{1,6,7}$ Tapping this potential requires a thorough investigation of the synthetically relevant properties of such enzymes, like substrate specificity, stereoselectivity, stability, and reaction scalability. ${ }^{8}$ Comprehensive studies on these enzymes are often complicated by high investment into the stereoselective synthesis of complex precursor surrogates and a significant number of analogues.

Chiral saturated oxygen heterocycles $(\mathrm{CSOH})$ are abundant, often pharmacophoric, structural elements of natural products with a strong influence on the molecular shape (Figure 1a).,10 Their selective synthesis is often hampered by a multitude of densely packed stereocenters. ${ }^{11}$ The intramolecular oxaMichael addition (IMOMA) basically provides access to $\mathrm{CSOH}$ with adjacent stereocenters starting from $\alpha$-branched precursors like 4 (Figure $1 \mathrm{~b}) .^{11-13}$

Chemical IMOMA, often integrated into efficient tandem processes, gained growing attention for the stereoselective synthesis of tetrahydropyrans (THPs) during recent years. $^{12-15}$ It has been shown that the cis- or trans-THP selectivity of IMOMA can be influenced by kinetic or thermodynamic reaction control, by the type of activation (acid, base, or metal catalysis), by the configuration of the double bond, and by the nature of the acceptor moiety. ${ }^{16-21}$ Although the reaction outcome is predictable for some systems (tandem-cross metathesis-IMOMA with vinylketone inter-

Received: November 22, 2019

Revised: March 20, 2020

Published: March 30, 2020

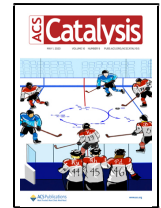




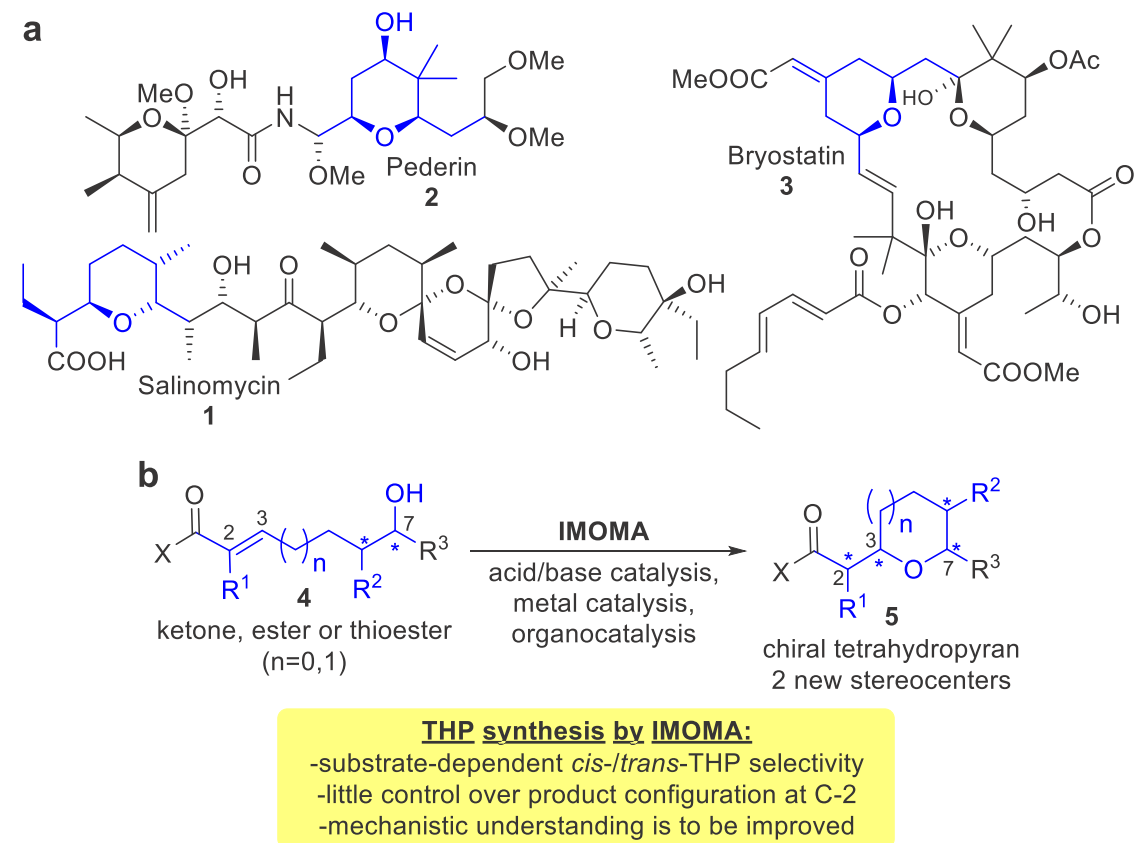

Figure 1. (a) Pharmacologically relevant natural products containing THPs, which are biosynthesized by IMOMA. (b) IMOMA enables stereoselective synthesis of chiral THPs and other CSOH. A C-2-C-6-bisbranched precursor is exemplarily shown.

Scheme 1. (a) AmbDH3 Catalyzes THP Formation in Ambruticin Biosynthesis. (b) Enzymatic In Vitro Studies on AmbDH3. $\mathrm{N}$-Acetylcysteamine (SNAC) Mimics the Natural Acyl Carrier Protein (ACP) Attachment. ${ }^{44,45}$
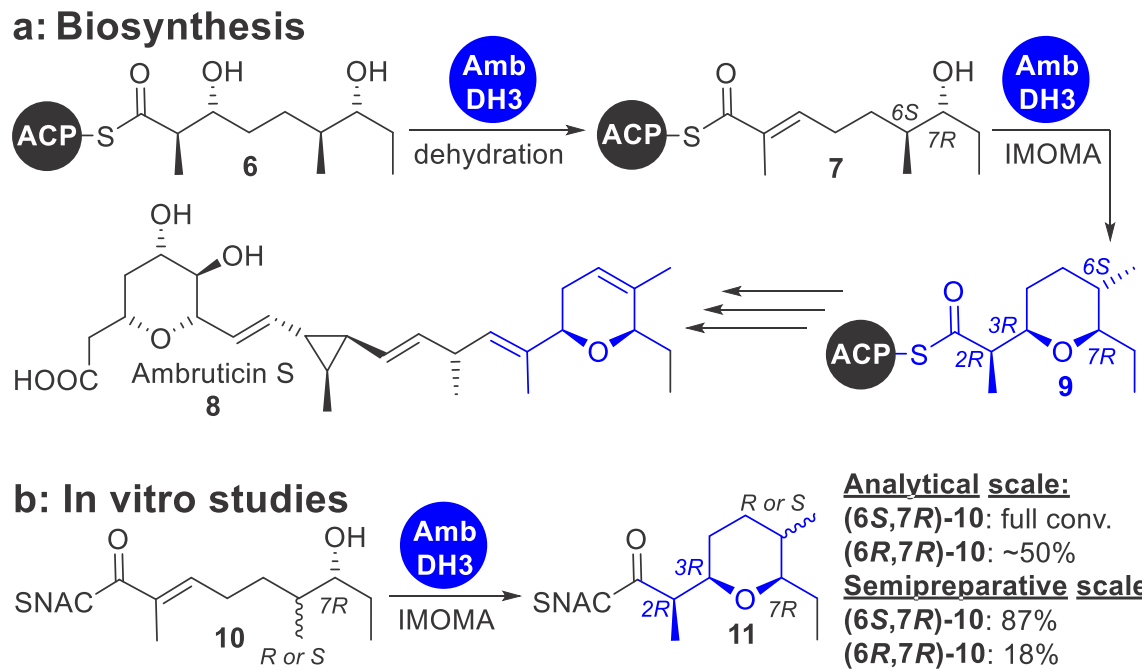

Analytical scale:

$(6 S, 7 R)-10$ : full conv.

$(6 R, 7 R)-10: \sim 50 \%$

Semipreparative scale:

$(6 S, 7 R)-10: 87 \%$

$(6 R, 7 R)-10: 18 \%$

mediates for example reliably leads to cis-THPs with diastereomeric excess > 5:1), ${ }^{15}$ low to moderate d.e.s are rather common and have only occasionally been improved by extensive reaction optimization or product re-equilibration (see, e.g., refs 22-27). Pioneering studies on the thioester IMOMA have provided valuable insight into the reasons for the high substrate dependence of the cis-trans selectivity, along with the establishment of guidelines for diastereoselective THP cyclization in specific systems. $^{16-19}$

Another important issue is the stereocontrol on C-2 of $\alpha$ branched Michael acceptors. The product configuration at this position is highly dependent on the molecular environment. ${ }^{13,28-30}$ Selectivity has been realized in cases when C-2 was incorporated into rings containing existing stereocenters. $^{31,32}$ The behavior of noncyclic systems is scarcely described (nearly all of these systems in the literature are not $\alpha$-branched). Multistep sequences currently used for the reliable synthesis of chiral THPs with adjacent stereocenters. $^{33,34}$ Taken together, the modest stereoselectivity of IMOMA combined with the harsh reaction conditions currently prevent the more frequent use of this reaction in organic synthesis. ${ }^{16}$ Novel variants that merge stereoselectivity and broad applicability are highly desirable. C-2 stereoselectivity and the potential to discriminate between $\mathrm{C}-7$ enantiomers/epimers would, for example, add valuable new features to the existing IMOMA methodology. Enzymatic catalysis with its tight control over extended transition states is particularly well-suited to provide a solution for the problems described above.

The biosynthesis of $\mathrm{CSOH}$ occurs by direct oxidation, by reduction of hemiacetals, or by intramolecular addition of hydroxyls to electrophiles. ${ }^{5,35,36}$ The synthetic applicability of 
Table 1. Substrate Surrogates That Were Reacted with Purified AmbDH3

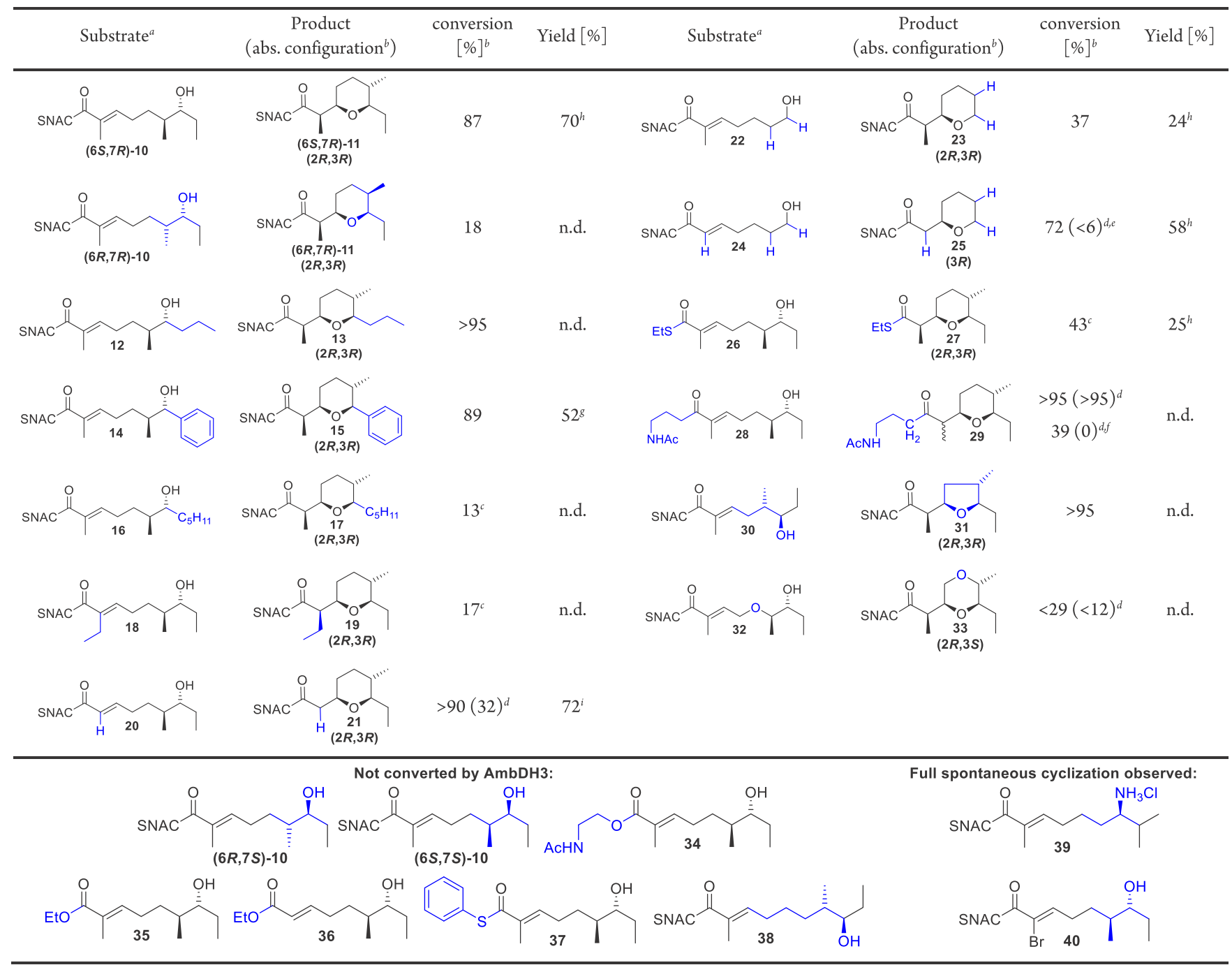

${ }^{a}$ Performed under nonoptimized conditions (see Supporting Information). ${ }^{b}$ Determined by ${ }^{1} \mathrm{H}$ NMR spectroscopy. ${ }^{c}$ Conducted on the $1-4$ mg scale with a substrate concentration of $2-4 \mathrm{mM}$ due to solubility issues. ${ }^{d}$ If applicable, the conversion of the spontaneous IMOMA under standard reaction conditions is shown in brackets. ${ }^{e}$ An e.e. of more than $95 \%$ was determined by ${ }^{1} \mathrm{H}$ NMR spectroscopy after reduction and esterification with $(S)$-Mosher's acid chloride. ${ }^{f}$ Conducted in an Eppendorf tube under shaking to suppress spontaneous cyclization of $\mathbf{2 8}$ that occurred during the stirred reaction. The d.e. and absolute configuration of the components in the crude reaction product could not be determined. ${ }^{g}$ Purified by semipreparative high-performance liquid chromatography. ${ }^{h}$ Purified by flash chromatography on silica gel. ${ }^{i}$ Crude yield.

such cyclizing enzymes has been successfully studied in some cases. The bifunctional cytochrome P450 monooxygenase AurH introduces a tetrahydrofuran (THF) ring in (+)-aureothin biosynthesis by direct oxidation. Hertweck et al. have biochemically characterized this enzyme and successfully applied it in a total synthesis of the natural product as part of an elegant late-stage kinetic resolution process. ${ }^{37-39}$ The same group reported on a polyketide synthase (PKS) domain for vinylogous chain branching that gives rise to a $\delta$-lactone in rhizoxin biosynthesis. ${ }^{40}$ This enzyme was extensively studied regarding its synthetic properties and could, for example, be employed for the synthesis of hard-to-access medium-sized lactones. $^{41-43}$ Enzymes that catalyze IMOMA have been discovered in the secondary metabolism, ${ }^{4-54}$ and a basic biochemical characterization has been reported for three enzymes from the ambruticin, the pederin, and the sorangicin biosynthetic pathways. ${ }^{4-46,55}$ Despite their diverse sequencebased annotation, recent studies suggest that all these IMOMA cyclases share an enzymatic mechanism in which they activate the 7-hydroxyl group of the precursor by acid-base catalysis for attack onto the Michael acceptor. ${ }^{45,47,55}$ No in depthstudies on the biocatalytic potential of IMOMA cyclases are reported to date.

We previously characterized the unusual bifunctional PKS domain $\mathrm{AmbDH} 3$ by a combination of enzymatic in vitro experiments, X-ray crystal structure analysis, and site-selective mutagenesis (Scheme 1a). ${ }^{44,45}$ It catalyzes a cascade of dehydration and IMOMA of 6 to the chiral THP 9 via 7. In vitro conversion experiments of the purified domain with synthetic substrate surrogates showed quantitative, stereospecific transformation of the natural precursor surrogate $(6 S, 7 R)-10$ into $(2 R, 3 R, 6 S, 7 R)-11$ in an analytical scale experiment and $87 \%$ conversion on the semipreparative scale ( $8 \mathrm{mg} / 27 \mu \mathrm{mol}$ starting material, Scheme $1 \mathrm{~b}$ ). The C-6 epimer $(6 R, 7 R)-10$ was partially converted into $(2 R, 3 R, 6 R, 7 R)-11$, suggesting substrate tolerance of the enzyme. 
Table 2. Reaction of AmbDH3 with Stereoisomeric Mixtures of 41 and 10

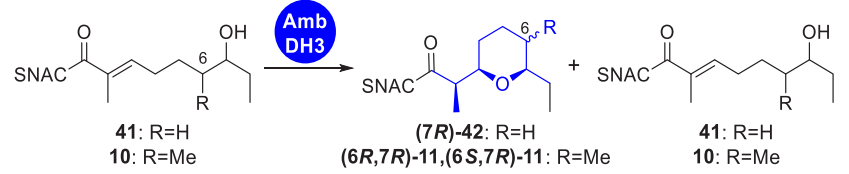

\begin{tabular}{|c|c|c|c|c|c|c|c|}
\hline $\mathrm{R}=\mathrm{H}$ & \multicolumn{4}{|c|}{ inserted stereoisomers of 41 [\%] } & conversion into $42^{b}$ & product ratio $^{b}$ & \\
\hline entry $^{a}$ & \multicolumn{2}{|c|}{$7 R$} & \multicolumn{2}{|c|}{$7 S$} & $S[(7 R)+(7 S)]$ & $(7 R) /(7 S)$ & \\
\hline 1 & 50 & \multicolumn{3}{|c|}{50} & $47 \%$ & $>95:<5$ & \\
\hline $\mathrm{R}=\mathrm{Me}$ & \multicolumn{4}{|c|}{ inserted stereoisomers of $\mathbf{1 0}[\%]$} & conversion into $11^{b}$ & \multicolumn{2}{|c|}{ product ratio $^{b}$} \\
\hline entry $^{a}$ & $6 S, 7 R$ & $6 R, 7 R$ & $6 S, 7 S$ & $6 R, 7 S$ & $\sum[(6 S, 7 R)+(6 R, 7 R)]$ & $(6 S, 7 R) /(6 R, 7 R)$ & $\mathrm{pr} / \mathrm{sr}^{\mathrm{c}}$ \\
\hline 2 & 50 & 0 & 0 & 50 & $41 \%$ & $>95:<5$ & n.d. \\
\hline 3 & 13 & 37 & 37 & 13 & $15 \%$ & $73: 27$ & 8.1 \\
\hline 4 & 30 & 30 & 30 & 10 & $32 \%$ & $90: 10$ & 9.1 \\
\hline 5 & 10 & 30 & 30 & 30 & $12 \%$ & $74: 26$ & 8.4 \\
\hline 6 & 26 & 24 & 24 & 26 & $23 \%$ & $90: 10$ & 8.5 \\
\hline
\end{tabular}

${ }^{a}$ The summed up concentration of the precursor stereoisomers was $2 \mathrm{mM}$ for 41 and $4 \mathrm{mM}$ for $10 .{ }^{b}$ The conversion of the individual stereoisomers of 10 was qualitatively estimated by analysis of the chiral high-performance liquid chromatography chromatograms. The overall conversion and the ratio of the cyclic stereoisomers in the conversions of 41 and 10 were quantified by ${ }^{1} \mathrm{H}$ NMR analysis in a separate experiment.

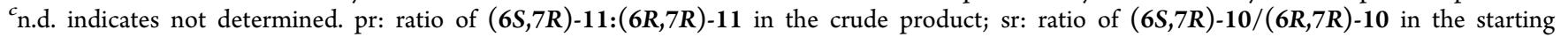
material.

\section{RESULTS AND DISCUSSION}

Substrate Specificity of AmbDH3. A library of precursor derivatives was stereoselectively synthesized via multistep sequences (see Supporting Information). For probing the substrate selectivity of $\mathrm{AmbDH} 3$, the members were individually incubated with the purified enzyme under the reported conditions $(8-10 \mathrm{mg} \approx 30 \mu \mathrm{mol}$ of starting material, Table 1, Figures S1-S26). ${ }^{44}$ Enzyme-free reference incubations determined the spontaneous background cyclization. The $7 S$-configured compounds $(6 R, 7 S)-10$ and $(6 S, 7 S)-10$ were not accepted, showing the crucial nature of the stereochemistry at C-7. Various alterations of the polyketide backbone substitution pattern were tolerated by the enzyme with the conversion increasing with the steric bulk of the residues on $\mathrm{C}$ $6 / \mathrm{C}-7$ (results for $(6 S, 7 R)-10,12,14$, and 22 ) and in the absence of electron-donating substituents on the acceptor (results for $(6 S, 7 R)-10,18,20,22$, and 24$)$. The low solubility of 16 and 18 in aqueous buffer prevented their reaction under standard assay conditions. Modest conversion was, however, observed in small-scale experiments of these compounds that were performed at lower concentration. The enzyme strongly differentiated between various types of carbonyls in the acceptor (results for $(6 S, 7 R)-10,26$, and 28 as well as 3437). Neither the dethia-oxo analogue 34 nor the other oxo esters $\mathbf{3 5}$ and $\mathbf{3 6}$ were competent substrates of AmbDH3. This underlines the aforementioned dependence of the enzymatic reaction on the electron density of the acceptor. Accordingly, the little soluble $S$-ethyl-enethioate $\mathbf{2 6}$ and vinylketone $\mathbf{2 8}$ were cyclized by the enzyme by $\sim 40 \%$ (see footnotes $c$, $d$, and $\mathrm{f}$ in Table 1). Phenylthioester $\mathbf{3 7}$ was not a competent substrate, showing that structural aspects play a role, even if the acceptor is sufficiently activated. While precursor $\mathbf{3 0}$ was fully converted by AmbDH3 into homochiral THF 31, oxepane precursor 38 was not a competent substrate. The decarba-oxo analogue 32 was partially converted into the 1,4-dioxane 33 . Substrates 39 and $\mathbf{4 0}$ underwent complete spontaneous cyclization under all tested reaction conditions, avoiding their assessment in this study.
Taken together, these results demonstrate a relaxed substrate specificity of AmbDH3. This might be explained by its origin as a modular type I PKS domain. The high relevance of protein-protein interactions in these systems goes along with lower substrate affinity and lower reaction rates of their individual domains compared to free enzymes. ${ }^{56-59}$ Indeed, the kinetic parameters for the reaction between $\mathrm{AmbDH} 3$ and $(6 S, 7 R)-10$ were determined to be in the range of previously observed values for reductive loop domains from modular type I PKS $\left(K_{\mathrm{m}}=8.2 \pm 3.1 \mathrm{mM}, k_{\text {cat }}=230 \pm 83 \mathrm{~s}^{-1}, k_{\text {cat }} / K_{\mathrm{m}}=28.2\right.$ $\pm 14.8 \mathrm{~s}^{-1} \times \mathrm{M}^{-1}$; Figure S28). ${ }^{56-59}$ A semipreparative scale time course experiment showed a steady and finally quantitative conversion in an overnight incubation, confirming that the rather slow reaction is not an issue at all for the use of the enzyme in biocatalytic reactions (Figures S29-S33). Only $2 R, 3 R$-/3R-configured products (or equivalent, like $(2 R, 3 S$ )33) were observed in the substrate specificity study, showing that the stereoselectivity of $\mathrm{AmbDH} 3$ is conserved among a broad range of substrates. The enzymatic cyclization gives access to various types of heterocycles, such as THFs (31) and 1,4-dioxanes (33). The low solubility of substrates like 16, 18, and 26 in aqueous buffer was identified as a cause for their low to modest conversion. This problem might be overcome by adaption of the reaction conditions (vide infra).

Molecular Modeling. After we had gained a comprehensive overview of the AmbDH3 substrate specificity, we turned to link this knowledge with structural data. Some members of the precursor library were modeled into the active site of the previously determined X-ray structure of AmbDH3 (PDB ID: $5 \mathrm{O} 15),{ }^{45}$ and the distances between $\mathrm{C}-3,7-\mathrm{OH}$ and the catalytic residues were put in relation to the conversion (Figure S27 and Table S1). A good correlation of the latter with the D215-7-OH distance was observed. Complex model structures of $\mathrm{AmbDH} 3$ with the noncompetent substrates $(6 S, 7 S)-10,37$ and 38 showed distances of more than $5 \AA$ and a bent backbone conformation that orients $7-\mathrm{OH}$ away from D215 so that its activation for a nucleophilic attack is prevented. In the complex models of all accepted substrates, these values were in the range of 1.9-3 $\AA$. Among these, a strict proportionality between distance and in vitro conversion 
Table 3. Optimization of the Reaction between (6S,7R)-10 and AmbDH3

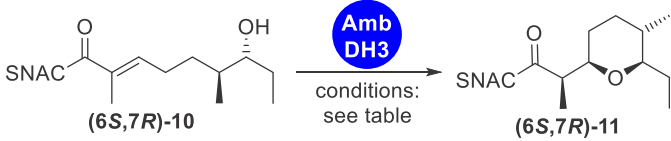

\begin{tabular}{|c|c|c|c|c|c|c|c|c|c|c|c|}
\hline \multicolumn{5}{|c|}{ conversion optimization $^{a}$} & \multicolumn{7}{|c|}{ yield optimization } \\
\hline entry & scale $[\mathrm{mg}]$ & enzyme $^{b}$ & cosolvent $^{c}$ & conversion $^{d}[\%]$ & entry & scale $[\mathrm{mg}]$ & $\mathrm{PhMe}[\mathrm{vol} \%]$ & $T\left[{ }^{\circ} \mathrm{C}\right]$ & $c$ (substrate) $[\mathrm{mM}]$ & workup $^{e}$ & yield $^{f}[\%]$ \\
\hline 1 & 10 & purified & & 87 & $1^{g}$ & 50 & $10 \%$ & 37 & 8 & A & 26 \\
\hline 2 & 30 & purified & & $<50$ & 2 & 50 & $10 \%$ & 37 & 4 & A & 60 \\
\hline 3 & 10 & lysate & & $>95$ & 3 & 50 & $10 \%$ & 37 & 2 & A & 54 \\
\hline 4 & 30 & lysate & & 93 & 4 & 50 & $5 \%$ & 37 & 4 & A & 66 \\
\hline 5 & 50 & lysate & & $<50$ & 5 & 50 & $2 \%$ & 37 & 4 & A & 68 \\
\hline 6 & 10 & lysate & $\mathrm{PhMe}$ & quant & 6 & 50 & $1 \%$ & 37 & 4 & A & 76 \\
\hline 7 & 30 & lysate & $\mathrm{PhMe}$ & $>95$ & 7 & 100 & $1 \%$ & 37 & 4 & A & 62 \\
\hline \multirow[t]{5}{*}{8} & 50 & lysate & $\mathrm{PhMe}$ & 94 & 8 & 100 & $1 \%$ & 30 & 4 & A & 66 \\
\hline & & & & & 9 & 100 & $1 \%$ & 25 & 4 & A & 64 \\
\hline & & & & & 10 & 50 & $1 \%$ & 30 & 4 & B & 80 \\
\hline & & & & & 11 & 50 & $1 \%$ & 30 & 4 & $\mathrm{C}$ & 80 \\
\hline & & & & & 12 & 50 & $1 \%$ & 30 & 4 & $\mathrm{D}$ & 76 \\
\hline
\end{tabular}

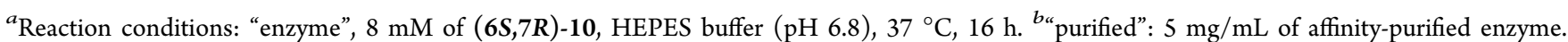
"lysate": The equivalent amount of cell pellet as for "purified" ( $2 \mathrm{~g}$ pellet $/ 50 \mathrm{mg}$ substrate) was sonicated, and the supernatant was used directly after centrifugation. ${ }^{c}$ The substrate was dissolved in the organic cosolvent and added to the cell lysate ( 10 vol\% cosolvent). ${ }^{d}$ The conversion was determined by ${ }^{1} \mathrm{H}$ NMR spectroscopy. ${ }^{e}$ Workup comprised triple extraction of the crude reaction mixture with EtOAc (A), accompanied by previous treatment with $\mathrm{CaCl}_{2}$ and proteinase $\mathrm{K}(\mathrm{B}), \mathrm{CaCl}_{2}$ and papain $(\mathrm{C})$, or $\mathrm{CaCl}_{2}$, proteinase $\mathrm{K}$, and papain $(\mathrm{D})$, respectively. ${ }^{f}$ The yield was determined after column chromatography on silica gel. ${ }^{g}$ Identical conditions as in entry 8 of "Conversion optimization".
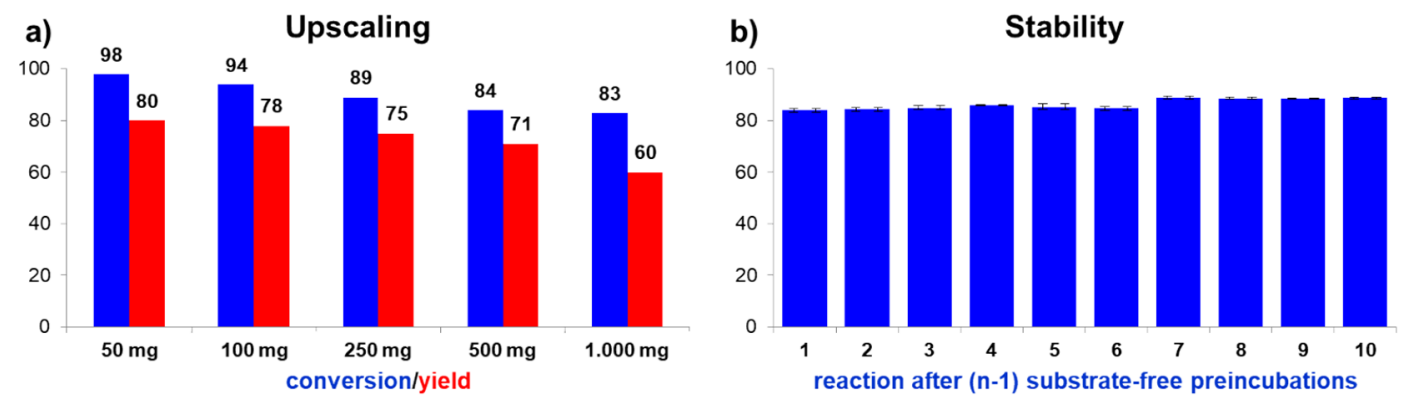

Figure 2. (a) Upscaling of the reaction to the preparative scale (conditions as in Table 3, right, entry 10). (b) Conversion of (6S,7R)-10 by $\mathrm{AmbDH} 3$ after various substrate-free preincubations of the enzyme.

was not observed, because other effects, like the low solubility of 16, 18, and 26, interfered. These results suggest that molecular modeling can be used to estimate the principle acceptance of substrates by $\mathrm{AmbDH} 3$. They also set the stage for a structure-guided directed evolution of AmbDH3 features.

Kinetic Resolution. The ability to efficiently differentiate between various presented stereoisomers forms the basis for Dynamic Kinetic Resolution (DKR) and Deracemization (DR). ${ }^{60,61} \mathrm{AmbDH} 3$ was incubated with various stereoisomeric mixtures of $\mathbf{4 1}$ and $\mathbf{1 0}$ (Table 2). Of rac-41, AmbDH3 cyclized only (7R)-41, while (7S)-41 remained unreacted (entry 1, Figures S34, S41, and S42). In incubations of $\mathrm{AmbDH} 3$ with various stereoisomer mixtures of 10, only $(6 S, 7 R)-10$ and $(6 R, 7 R)-10$ were converted into the expected cis-THPs, albeit to a strongly varying degree (entries 2-6, Figures S35-S40 and S43-S47). (6S,7R)-10 was converted in all reactions by at least $80 \%$, irrespective of the ratio of competing stereoisomers. $(6 S, 7 R)-11$ was the major stereoisomer formed in all reactions with $(6 S, 7 R)-11 /(6 R, 7 R)-11$ ratios of up to $90: 10$.

This selectivity opens the possibility to develop novel types of DKR or DR processes to chiral $\mathrm{CSOH}$ that would provide control over up to four stereocenters. Dynamic epimerization of the secondary alcohol could be achieved by adapting established systems for chemoenzymatic DKR using transfer hydrogenation catalysts or by alcohol dehydrogenase-catalyzed $\mathrm{DR}^{62-70}$ Under ketone-epimerizing conditions, the stereocenter at C-6 could additionally be included.

Upscaling of the Enzymatic Reaction. Lack of scalability is a common problem of enzymatic transformations. To enable the preparative-scale synthesis of chiral THP building blocks, we performed an optimization of the cyclase reaction conditions (Table 3). Scaling up the reaction between AmbDH3 and (6S,7R)-10 under the previously described conditions led to a significant decline in conversion for reaction batches above $10 \mathrm{mg}(33.3 \mu \mathrm{mol})$ starting material (left, entries 1 and 2). ${ }^{44}$ An upscaling to $50 \mathrm{mg}$ batches with a conversion of $94 \%$ became possible by using the cell-free extract instead of the isolated enzyme and by adding $10 \%$ of toluene to the reaction (entries 3-8). Reducing the substrate concentration from 8 to $4 \mathrm{mM}$ and fine-tuning of the solvent amount gave $76 \%$ yield on the $50 \mathrm{mg}$ scale (Table 3, right, entries 1-6). At higher quantities, increased precipitation of the enzyme led to lower yield (entry 7 ). This problem was 
Scheme 2. Chemoenzymatic Total Synthesis of (-)-centrolobine ${ }^{a}$

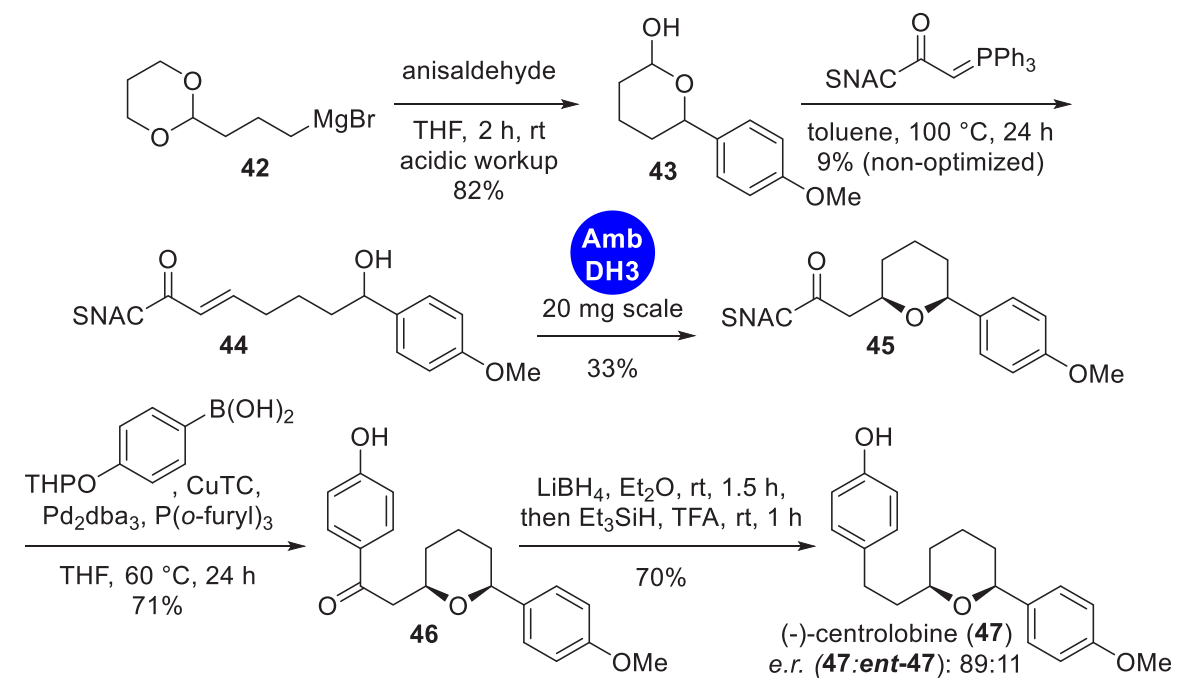

${ }^{a}$ Using AmbDH3 for cis-THP-formation in the key step.

addressed by reducing the reaction temperature and by treating with a proteinase before extractive workup (entries $7-12)$. An isolated yield of $80 \%$ was reproducibly obtained on the $50 \mathrm{mg}$ scale along with nearly complete recovery of the inserted organic material (entries 10 and 11). The established conditions allowed a further upscaling to batches of $1 \mathrm{~g}(3.32$ mmol) starting material (Figure 2a, Figure S48). $83 \%$ Conversion and $60 \%$ isolated yield $(15 \%$ recovery of $(6 S, 7 R)-10)$ after one round of workup were observed. Further recovery of organic material was possible after repetitive proteinase treatment of the precipitate. The scalability and clean course of the reaction shows that this enzymatic transformation is suitable for producing preparative batches of chiral heterocyclic building blocks under operationally simple conditions.

Stability of AmbDH3. To investigate the stability of the enzyme, we reacted $\mathrm{AmbDH} 3$ with $(6 S, 7 R)-10$ after repetitive, substrate-free exhaustion cycles (Figure 2b, Figures S49-S58). The enzyme was incubated up to nine times in the absence of substrate for $16 \mathrm{~h}$ at $30^{\circ} \mathrm{C}$, interrupted by resting periods of 8 $\mathrm{h}$ at $4{ }^{\circ} \mathrm{C}$. In the respective ultimate cycle, $(6 S, 7 R)-10$ was added, and the conversion was determined after $16 \mathrm{~h}$ of reaction. The conversion remained on a high level independent of the number of substrate-free preincubations. Lyophilization or freeze-thawing of $\mathrm{AmbDH} 3$ also had no detrimental effect on the conversion (Figures S59-S68 and Tables S2 and S3).

Chemoenzymatic Total Synthesis. Pyran-containing diarylheptanoids are plant natural products with antibiotic, antiprofilerative, and antioxidative activity. ${ }^{71,72}(-)$-Centrolobine (47) is a member of this class that shows no further structural similarities to the ambruticins and thus represents an optimal model system to demonstrate the suitability of $\mathrm{AmbDH} 3$ for general chemoenzymatic synthesis (Scheme 2, for reported total syntheses see, e.g., refs 73-76). Racemic 44 was synthesized in two steps from Grignard reagent 42. Enzymatic cyclization of $\mathbf{4 4}$ on a reaction scale of $20 \mathrm{mg}$ (57 $\mu \mathrm{mol}$ ) starting material resolved the enantio-enriched syn-THP with a conversion of $40 \%$ and an isolate yield of $33 \%$. The $\mathrm{N}$-acetylcysteamine (SNAC) moiety was exchanged with a 4-hydroxyphenyl group by Liebeskind-Srogl coupling. Ketone reduction using $\mathrm{LiBH}_{4}$ followed by $\mathrm{EtSiH}_{3}$ and TFA treatment led to a mixture of (-)-centrolobine (46) and (+)-centrolobine (ent-46). The enantiomeric ratio of $89: 11$ in favor of 46 suggests that AmbDH3 exhibits comparable selectivity for the precursor (7R)-44 as for the natural substrate surrogate (see section "Kinetic Resolution"). The substrate tolerance and stereoselectivity as well as the scalability of the AmbDH3 reactions supersede the need for any further stereoselective chemical transformations.

\section{CONCLUSION}

We report our investigations on $\mathrm{AmbDH} 3$, a member of the recently discovered, but biocatalytically not exploited, group of IMOMA cyclases. AmbDH3 exhibits relaxed substrate specificity and conserved stereoselectivity along a broad range of substrates, like enethioates and vinylketones, providing access to various types of $\mathrm{CSOH}$ like THPs, THFs, and 1,4-dioxanes. The high stereoselectivity of $\mathrm{AmbDH} 3$ on $\mathrm{C}-2$ and the efficient discrimination between individual C-6 and C-7 stereoisomers go beyond the scope of the current synthetic methodology for IMOMA. These features were exploited in the resolution of chiral THPs with up to four asymmetric centers from racemic precursor mixtures and might form the basis for the development of multienzyme or hybrid catalytic systems for DKR or DR to CSOH. Upscaling of the enzymatic cyclization succeeded, providing access to preparative amounts of chiral THP. The enzyme was successfully applied in the enantioselective total synthesis of the THP natural product (-)-centrolobine.

This work is one of the rare examples in which isolated PKS domains were investigated regarding their performance in (semi)preparative enzymatic synthesis. Future work will aim to improve the reaction conditions to expand the scope to hydrophobic substrates and more common Michael acceptors like $S$-alkylthioesters and vinylketones. The reported AmbDH3 structure will form a solid base for improving relevant properties by enzyme engineering. ${ }^{77}$ Our work paves the way for the characterization of IMOMA cyclases from other biosynthetic pathways to expand the scope of biocatalysts and accessible $\mathrm{CSOH}$ types. Incorporation of IMOMA cyclases into (chemo)enzymatic cascades might expand the product spectrum to other types of chiral heterocycles like piperidines. 
Finally, the combined results on the AmbDH3 structurefunction relationship and its substrate specificity profile are an important milestone on the way to a rational engineering of cyclase activities into existing multimodular assembly lines in the frame of directed biosynthesis approaches. ${ }^{45}$

\section{EXPERIMENTAL SECTION}

Enzyme Expression and Purification. The plasmid $a m b D H 3 \_$ET28a(+) was transformed into chemically competent E. coli BL21(DE3) cells. A single colony was used for inoculation in dYT medium with kanamycin (final concentration $0.1 \mathrm{mM}$ ) for plasmid selection. Cultures were grown to an $\mathrm{OD}_{600}$ of 0.5 at $37^{\circ} \mathrm{C}$, induced with $0.1 \mathrm{mM}$ isopropyl $\beta$-D1-thiogalactopyranoside, and shaken at $15{ }^{\circ} \mathrm{C}$ for $22 \mathrm{~h}$. Cells were harvested by centrifugation $\left(5000 \mathrm{~g}, 4{ }^{\circ} \mathrm{C}, 15 \mathrm{~min}\right)$. For purification, $1 \mathrm{~g}$ of cells was suspended in $10 \mathrm{~mL}$ of 4-(2hydroxyethyl)-1-piperazineethanesulfonic acid (HEPES) buffer ( $\mathrm{pH}$ 6.8, $25 \mathrm{mM}$ HEPES, $100 \mathrm{mM} \mathrm{NaCl}$ ), and cell disruption was performed on ice by sonication ( $45 \%$ amplitude, 10 cycles, $0.02 \mathrm{~kJ} / \mathrm{s}, 30 \mathrm{~s}$ sonication, $30 \mathrm{~s}$ pause). After centrifugation $\left(10000 \mathrm{~g}, 4^{\circ} \mathrm{C}, 30 \mathrm{~min}\right)$ the obtained crude lysate was filtered (0.45 $\mu \mathrm{m}$, cellulose acetate), and the target protein was purified by fast protein liquid chromatography (FPLC) equipped with a HisTrap FF Ni-NTA column. The column was washed with washing buffer $(30 \mathrm{mM}$ Tris, $500 \mathrm{mM} \mathrm{NaCl}$, $10 \mathrm{mM}$ imidazole, $10 \%$ glycerol, $\mathrm{pH} 7.5$ ). Elution of the target protein could be observed during a linear gradient elution with elution buffer $(30 \mathrm{mM}$ Tris, $500 \mathrm{mM} \mathrm{NaCl}, 500 \mathrm{mM}$ imidazole, $10 \%$ glycerol, $\mathrm{pH} 7.5$ ) from 0 to $100 \%$ in $25 \mathrm{~mL}$. The protein-containing fractions were united, and the elution buffer was replaced by HEPES buffer (25 mM HEPES, 100 $\mathrm{mM} \mathrm{NaCl}, \mathrm{pH}$ 6.8) using $\mathrm{PD}-10$ desalting column. If necessary, the purified enzyme was concentrated and immediately used for activity assays.

Semipreparative Scale Conversions. Assays for determination of substrate tolerance were performed in a total volume of $4 \mathrm{~mL}$ containing $8-10 \mathrm{mM}$ of substrate and 5.0-5.4 $\mathrm{mg} / \mathrm{mL}$ of AmbDH3 in HEPES buffer ( $\mathrm{pH} 6.8,25 \mathrm{mM}$ HEPES, $100 \mathrm{mM} \mathrm{NaCl}$ ). After incubation at $37^{\circ} \mathrm{C}$ for $16 \mathrm{~h}$, the sample was extracted with $3 \times 5 \mathrm{~mL}$ ethyl acetate (EtOAc), and the solvent was removed under reduced pressure. The product was dissolved in $\mathrm{CDCl}_{3}$ and analyzed by ${ }^{1} \mathrm{H}$ NMR spectroscopy to determine the conversion.

Preparative-Scale Enzymatic Conversions. For preparative-scale assays, $2.0 \mathrm{~g}$ cell pellet per $50 \mathrm{mg}$ substrate were resolved in HEPES buffer ( $1 \mathrm{~mL}$ per $0.1 \mathrm{~g}$ pellet, $25 \mathrm{mM}$ HEPES, $100 \mathrm{mM} \mathrm{NaCl}, \mathrm{pH} 6.8$ ), and cells were disrupted by sonication (45\% amplitude, 10 cycles, $0.02 \mathrm{~kJ} / \mathrm{s}, 30 \mathrm{~s}$ sonication, $30 \mathrm{~s}$ pause). After centrifugation $(10000 \mathrm{~g}, 30$ min, $4{ }^{\circ} \mathrm{C}$ ), the crude lysate was directly used for the enzyme assays without further purification. The substrate was dissolved in toluene ( $1 \mathrm{vol} \%)$, the volume was filled up to give a final substrate concentration of $4 \mathrm{mM}$, and the crude lysate was added. After it was stirred at $30{ }^{\circ} \mathrm{C}$ and $175 \mathrm{rpm}$ for $16 \mathrm{~h}$, proteinase $\mathrm{K}$ (4 mg per $50 \mathrm{mg}$ substrate) and $\mathrm{CaCl}_{2}$ (final concentration $1 \mathrm{mM}$ ) were added, and the reaction was stirred at $37{ }^{\circ} \mathrm{C}$ for $2 \mathrm{~h}$. The phases were separated, and the aqueous phase was extracted with EtOAc. The combined organic phases were washed with saturated $\mathrm{NaCl}$ solution, dried over $\mathrm{MgSO}_{4}$, and concentrated under reduced pressure. The conversion was determined by integration of the appropriate signals in the ${ }^{1} \mathrm{H}$ NMR spectrum. Purification by flash chromatography on silica gel $\left(\mathrm{CH}_{2} \mathrm{Cl}_{2} /\right.$ EtOAc/2:1 $\left.\rightarrow 0: 1\right)$ gave the product $(6 S, 7 R)-11$ as colorless solid. Variations of this procedure were implemented as described in Table 3.

\section{ASSOCIATED CONTENT}

Supporting Information

The Supporting Information is available free of charge at https://pubs.acs.org/doi/10.1021/acscatal.9b05071.

Methods and materials, synthetic procedures, studies on the substrate tolerance of $\mathrm{AmbDH} 3$, docking calculations, kinetic analysis, time course experiment, kinetic resolution, upscaling of the enzymatic reaction, stability studies (PDF)

NMR spectra for all synthetic compounds and assay products (PDF)

\section{AUTHOR INFORMATION}

\section{Corresponding Author}

Frank Hahn - Centre for Biomolecular Drug Research, Leibniz Universität Hannover, 30167 Hannover, Germany; Professur für Organische Chemie (Lebensmittelchemie), Fakultät für Biologie, Chemie und Geowissenschaften, Department of Chemistry, Universität Bayreuth, 95447 Bayreuth, Germany; ○ orcid.org/0000-0002-0603-3482; Email: frank.hahn@unibayreuth.de

\section{Authors}

Tim Hollmann - Professur für Organische Chemie (Lebensmittelchemie), Fakultät für Biologie, Chemie und Geowissenschaften, Department of Chemistry, Universität Bayreuth, 95447 Bayreuth, Germany

Gesche Berkhan - Centre for Biomolecular Drug Research, Leibniz Universität Hannover, 30167 Hannover, Germany; Professur für Organische Chemie (Lebensmittelchemie), Fakultät für Biologie, Chemie und Geowissenschaften, Department of Chemistry, Universität Bayreuth, 95447 Bayreuth, Germany

Lisa Wagner - Professur für Organische Chemie (Lebensmittelchemie), Fakultät für Biologie, Chemie und Geowissenschaften, Department of Chemistry, Universität Bayreuth, 95447 Bayreuth, Germany

Kwang Hoon Sung - Helmholtz-Zentrum für Infektionsforschung GmbH, 38124 Braunschweig, Germany; Institute of Biochemistry, Biotechnology and Bioinformatics, Technische Universität Braunschweig, 38106 Braunschweig, Germany; Protein Facility, ILAb Co., Ltd. NP513, The Catholic University of Korea, 420-743 Bucheon, Republic of Korea

Simon Kolb - Professur für Organische Chemie (Lebensmittelchemie), Fakultät für Biologie, Chemie und Geowissenschaften, Department of Chemistry, Universität Bayreuth, 95447 Bayreuth, Germany

Hendrik Geise - Centre for Biomolecular Drug Research, Leibniz Universität Hannover, 30167 Hannover, Germany

Complete contact information is available at:

https://pubs.acs.org/10.1021/acscatal.9b05071

\section{Author Contributions}

${ }^{\#}$ T.H. and G.B.: These authors contributed equally to this work.

Notes

The authors declare no competing financial interest. 


\section{ACKNOWLEDGMENTS}

Funding from the Emmy Noether program of the DFG (HA 5841/2-1), a Sachbeihilfe of the DFG (HA 5841/4-1), the Marie Curie program of the European Union (Project No. 293430), the Graduate School MINAS (doctoral stipend to G.B.), and the Deutsche Bundesstiftung Umwelt (doctoral stipend to L.W.) are gratefully acknowledged. We thank the MS facilities and NMR facilities of the OCI Hannover and the Faculty BCG, Univ. of Bayreuth, as well as the Nordbayerisches NMR-Zentrum.

\section{DEDICATION}

This paper is dedicated to Prof. Peter F. Leadlay on the occasion of his recent retirement.

\section{REFERENCES}

(1) Friedrich, S.; Hahn, F. Opportunities for Enzyme Catalysis in Natural Product Chemistry. Tetrahedron 2015, 71, 1473-1508.

(2) Sattely, E. S.; Fischbach, M. A.; Walsh, C. T. Total Biosynthesis: In Vitro Reconstitution of Polyketide and Nonribosomal Peptide Pathways. Nat. Prod. Rep. 2008, 25, 757-793.

(3) Horsman, M. E.; Hari, T. P. A.; Boddy, C. N. Polyketide Synthase and Non-Ribosomal Peptide Synthetase Thioesterase Selectivity: Logic Gate or a Victim of Fate? Nat. Prod. Rep. 2016, 33, 183-202.

(4) Christianson, D. W. Structural and Chemical Biology of Terpenoid Cyclases. Chem. Rev. 2017, 117, 11570-11648.

(5) Hemmerling, F.; Hahn, F. Biosynthesis of Oxygen and NitrogenContaining Heterocycles in Polyketides. Beilstein J. Org. Chem. 2016, $12,1512-1550$.

(6) Turner, N. J.; O’Reilly, E. Biocatalytic Retrosynthesis. Nat. Chem. Biol. 2013, 9, 285-288.

(7) Yuzawa, S.; Zargar, A.; Pang, B.; Katz, L.; Keasling, J. D. Commodity Chemicals From Engineered Modular Type I Polyketide Synthases. In Methods in Enzymology; Scrutton, N., Ed.; Enzymes in Synthetic Biology; Academic Press: Amsterdam, Netherlands, 2018; Vol. 608, pp 393-415.

(8) Reetz, M. T. Biocatalysis in Organic Chemistry and Biotechnology: Past, Present, and Future. J. Am. Chem. Soc. 2013, 135, 12480-12496.

(9) Pozharskii, A. F.; Soldatenkov, A.; Katritzky, A. R. Heterocycles in Life and Society: An Introduction to Heterocyclic Chemistry, Biochemistry and Applications, 2nd ed.; Wiley-VCH: Weinheim, Germany, 2011.

(10) Taylor, R. D.; MacCoss, M.; Lawson, A. D. G. Rings in Drugs. J. Med. Chem. 2014, 57, 5845-5859.

(11) Cossy, J.; Guérinot, A. Natural Products Containing Oxygen Heterocycles-Synthetic Advances Between 1990 and 2015. In Advances in Heterocyclic Chemistry; Scriven, E. F. V., Ramsden, C. A., Eds.; Heterocyclic Chemistry in the 21st Century; Academic Press: Amsterdam, Netherlands, 2016; Vol. 119, pp 107-142.

(12) Nasir, N. M.; Ermanis, K.; Clarke, P. A. Strategies for the Construction of Tetrahydropyran Rings in the Synthesis of Natural Products. Org. Biomol. Chem. 2014, 12, 3323-3335.

(13) Nising, C. F.; Bräse, S. Recent Developments in the Field of Oxa-Michael Reactions. Chem. Soc. Rev. 2012, 41, 988-999.

(14) Hu, J.; Bian, M.; Ding, H. Recent Application of Oxa-Michael Reaction in Complex Natural Product Synthesis. Tetrahedron Lett. 2016, 57, 5519-5539.

(15) Sánchez-Roselló, M.; Miró, J.; del Pozo, C. Cross-Metathesis/ Intramolecular (Hetero-)Michael Addition: A Convenient Sequence for the Generation of Carbo- and Heterocycles. Synthesis 2017, 49, 2787-2802.

(16) Ermanis, K.; Hsiao, Y.-T.; Kaya, U.; Jeuken, A.; Clarke, P. A. The Stereodivergent Formation of 2,6-Cis and 2,6-Trans-Tetrahydropyrans: Experimental and Computational Investigation of the Mechanism of a Thioester Oxy-Michael Cyclization. Chem. Sci. 2017, $8,482-490$
(17) Clarke, P. A.; Ermanis, K. Synthesis of the C20-C32 Tetrahydropyran Core of the Phorboxazoles and the C22 Epimer via a Stereodivergent Michael Reaction. Org. Lett. 2012, 14, 55505553.

(18) Fuwa, H.; Noto, K.; Sasaki, M. Biosynthesis-Inspired Intramolecular Oxa-Conjugate Cyclization of $\alpha, \beta$-Unsaturated Thioesters: Stereoselective Synthesis of 2,6-Cis-Substituted Tetrahydropyrans. Org. Lett. 2011, 13, 1820-1823.

(19) Fuwa, H.; Ichinokawa, N.; Noto, K.; Sasaki, M. Stereoselective Synthesis of 2,6-Cis-Substituted Tetrahydropyrans: Brønsted AcidCatalyzed Intramolecular Oxa-Conjugate Cyclization of $\alpha, \beta$-Unsaturated Ester Surrogates. J. Org. Chem. 2012, 77 (6), 2588-2607.

(20) Schneider, C.; Schuffenhauer, A. Stereodivergent Synthesis of Highly Substituted Tetrahydropyrans. Eur. J. Org. Chem. 2000, 2000, $73-82$.

(21) Banwell, M. G.; Bui, C. T.; Pham, H. T. T.; Simpson, G. W. Michael Addition of $\mathrm{N}$ - and O-Centred Nucleophiles to Tethered Acrylates. The Role of Double Bond Geometry in Controlling the Diastereoselectivity of Cyclisations Leading to 2,6-Disubstituted Tetrahydropyrans and Piperidines. J. Chem. Soc., Perkin Trans. 1 1996, No. 10, 967-969.

(22) Bates, R. W.; Li, L.; Palani, K.; Phetsang, W.; Loh, J. K. Synthesis of the Tetrahydropyran Fragment of Bistramide D. Asian J. Org. Chem. 2014, 3, 792-796.

(23) Bates, R. W.; Song, P. Tetrahydropyran Synthesis by Intramolecular Conjugate Addition to Enones: Synthesis of the Clavosolide Tetrahydropyran Ring. Synthesis 2010, 2010, 2935-2942.

(24) Uenishi, J.; Iwamoto, T.; Tanaka, J. Total Synthesis of (-)-Zampanolide and Questionable Existence of (-)-Dactylolide as the Elusive Biosynthetic Precursor of (-)-Zampanolide in an Okinawan Sponge. Org. Lett. 2009, 11, 3262-3265.

(25) Wang, B.; Hansen, T. M.; Wang, T.; Wu, D.; Weyer, L.; Ying, L.; Engler, M. M.; Sanville, M.; Leitheiser, C.; Christmann, M.; Lu, Y.; Chen, J.; Zunker, N.; Cink, R. D.; Ahmed, F.; Lee, C.-S.; Forsyth, C. J. Total Synthesis of Phorboxazole A via de Novo Oxazole Formation: Strategy and Component Assembly. J. Am. Chem. Soc. 2011, 133, 1484-1505.

(26) Ferrié, L.; Boulard, L.; Pradaux, F.; Bouzbouz, S.; Reymond, S.; Capdevielle, P.; Cossy, J. Synthetic Efforts toward the Macrolactone Core of Leucascandrolide A. J. Org. Chem. 2008, 73, 1864-1880.

(27) Evans, D. A.; Ripin, D. H. B.; Halstead, D. P.; Campos, K. R. Synthesis and Absolute Stereochemical Assignment of (+)-Miyakolide. J. Am. Chem. Soc. 1999, 121, 6816-6826.

(28) Dong, C.-G.; Henderson, J. A.; Kaburagi, Y.; Sasaki, T.; Kim, D.-S.; Kim, J. T.; Urabe, D.; Guo, H.; Kishi, Y. New Syntheses of E7389 C14-C35 and Halichondrin C14-C38 Building Blocks: Reductive Cyclization and Oxy-Michael Cyclization Approaches. J. Am. Chem. Soc. 2009, 131, 15642-15646.

(29) Yoshida, M.; Takikawa, H.; Mori, K. Triterpenoid Total Synthesis. Part 6. Synthesis of Testudinariols A and B, Triterpene Metabolites of the Marine Mollusc Pleurobrancus Testudinarius. J. Chem. Soc., Perkin Trans. 1 2001, No. 9, 1007-1017.

(30) Tatsuta, K.; Nakano, S.; Narazaki, F.; Nakamura, Y. The First Total Synthesis and Establishment of Absolute Structure of Luminacins $\mathrm{C} 1$ and C2. Tetrahedron Lett. 2001, 42, 7625-7628.

(31) Li, Y.; Chen, Z.-X.; Xiao, Q.; Ye, Q.-D.; Sun, T.-W.; Meng, F.K.; Ren, W.-W.; You, L.; Xu, L.-M.; Wang, Y.-F.; Chen, J.-H.; Yang, Z. Diastereoselective Total Synthesis of $( \pm)$-Schindilactone A, Part 2: Construction of the Fully Functionalized CDEFGH Ring System. Chem. - Asian J. 2012, 7, 2334-2340.

(32) Coffey, D. S.; Overman, L. E.; Stappenbeck, F. Enantioselective Total Syntheses of 13,14,15-Isocrambescidin 800 and 13,14,15Isocrambescidin 657. J. Am. Chem. Soc. 2000, 122, 4904-4914.

(33) Godin, F.; Prévost, M.; Viens, F.; Mochirian, P.; Brazeau, J.-F.; Gorelsky, S. I.; Guindon, Y. A Study of Exocyclic Radical Reductions of Polysubstituted Tetrahydropyrans. J. Org. Chem. 2013, 78, 60756103.

(34) Godin, F.; Prévost, M.; Gorelsky, S. I.; Mochirian, P.; Nguyen, M.; Viens, F.; Guindon, Y. Diastereoselective Hydrogen-Transfer 
Reactions: An Experimental and DFT Study. Chem. - Eur. J. 2013, 19, 9308-9318.

(35) Wang, L.; Parnell, A.; Williams, C.; Bakar, N. A.; Challand, M. R.; van der Kamp, M. W.; Simpson, T. J.; Race, P. R.; Crump, M. P.; Willis, C. L. A Rieske Oxygenase/Epoxide Hydrolase-Catalysed Reaction Cascade Creates Oxygen Heterocycles in Mupirocin Biosynthesis. Nat. Catal. 2018, 1, 968.

(36) Meng, S.; Tang, G.-L.; Pan, H.-X. Enzymatic Formation of Oxygen-Containing Heterocycles in Natural Product Biosynthesis. ChemBioChem 2018, 19, 2002-2022.

(37) Werneburg, M.; Busch, B.; He, J.; Richter, M. E. A.; Xiang, L.; Moore, B. S.; Roth, M.; Dahse, H.-M.; Hertweck, C. Exploiting Enzymatic Promiscuity to Engineer a Focused Library of Highly Selective Antifungal and Antiproliferative Aureothin Analogues. J. Am. Chem. Soc. 2010, 132, 10407-10413.

(38) Henrot, M.; Richter, M. E. A.; Maddaluno, J.; Hertweck, C.; De Paolis, M. Convergent Asymmetric Synthesis of (+)-Aureothin Employing an Oxygenase-Mediated Resolution Step. Angew. Chem., Int. Ed. 2012, 51, 9587-9591.

(39) He, J.; Müller, M.; Hertweck, C. Formation of the Aureothin Tetrahydrofuran Ring by a Bifunctional Cytochrome P450 Monooxygenase. J. Am. Chem. Soc. 2004, 126, 16742-16743.

(40) Bretschneider, T.; Heim, J. B.; Heine, D.; Winkler, R.; Busch, B.; Kusebauch, B.; Stehle, T.; Zocher, G.; Hertweck, C. Vinylogous Chain Branching Catalysed by a Dedicated Polyketide Synthase Module. Nature 2013, 502, 124-128.

(41) Sundaram, S.; Kim, H. J.; Bauer, R.; Thongkongkaew, T.; Heine, D.; Hertweck, C. On-Line Polyketide Cyclization into Diverse Medium-Sized Lactones by a Specialized Ketosynthase Domain. Angew. Chem., Int. Ed. 2018, 57, 11223-11227.

(42) Heine, D.; Sundaram, S.; Bretschneider, T.; Hertweck, C. Twofold Polyketide Branching by a Stereoselective Enzymatic Michael Addition. Chem. Commun. 2015, 51, 9872-9875.

(43) Heine, D.; Bretschneider, T.; Sundaram, S.; Hertweck, C. Enzymatic Polyketide Chain Branching To Give Substituted Lactone, Lactam, and Glutarimide Heterocycles. Angew. Chem., Int. Ed. 2014, 53, 11645-11649.

(44) Berkhan, G.; Hahn, F. A Dehydratase Domain in Ambruticin Biosynthesis Displays Additional Activity as a Pyran-Forming Cyclase. Angew. Chem., Int. Ed. 2014, 53, 14240-14244.

(45) Sung, K. H.; Berkhan, G.; Hollmann, T.; Wagner, L.; Blankenfeldt, W.; Hahn, F. Insights into the Dual Activity of a Bifunctional Dehydratase-Cyclase Domain. Angew. Chem., Int. Ed. 2018, 57, 343-347.

(46) Pöplau, P.; Frank, S.; Morinaka, B. I.; Piel, J. An Enzymatic Domain for the Formation of Cyclic Ethers in Complex Polyketides. Angew. Chem., Int. Ed. 2013, 52, 13215-13218.

(47) Luhavaya, H.; Dias, M. V. B.; Williams, S. R.; Hong, H.; de Oliveira, L. G.; Leadlay, P. F. Enzymology of Pyran Ring A Formation in Salinomycin Biosynthesis. Angew. Chem., Int. Ed. 2015, 54, 1362213625 .

(48) Irschik, H.; Kopp, M.; Weissman, K. J.; Buntin, K.; Piel, J.; Müller, R. Analysis of the Sorangicin Gene Cluster Reinforces the Utility of a Combined Phylogenetic/Retrobiosynthetic Analysis for Deciphering Natural Product Assembly by Trans-AT PKS. ChemBioChem 2010, 11, 1840-1849.

(49) Li, C.; Roege, K. E.; Kelly, W. L. Analysis of the Indanomycin Biosynthetic Gene Cluster from Streptomyces Antibioticus NRRL 8167. ChemBioChem 2009, 10, 1064-1072.

(50) Woo, A. J.; Strohl, W. R.; Priestley, N. D. Nonactin Biosynthesis: The Product of NonS Catalyzes the Formation of the Furan Ring of Nonactic Acid. Antimicrob. Agents Chemother. 1999, 43, 1662-1668.

(51) Rebets, Y.; Brötz, E.; Manderscheid, N.; Tokovenko, B.; Myronovskyi, M.; Metz, P.; Petzke, L.; Luzhetskyy, A. Insights into the Pamamycin Biosynthesis. Angew. Chem., Int. Ed. 2015, 54, 22802284.
(52) Matilla, M. A.; Stöckmann, H.; Leeper, F. J.; Salmond, G. P. C. Biogenesis of the Broad Spectrum Antifungal and Antioomycete Compound, Oocydin A. J. Biol. Chem. 2012, 287, 39125-39138.

(53) Sudek, S.; Lopanik, N. B.; Waggoner, L. E.; Hildebrand, M.; Anderson, C.; Liu, H.; Patel, A.; Sherman, D. H.; Haygood, M. G. Identification of the Putative Bryostatin Polyketide Synthase Gene Cluster from "Candidatus Endobugula Sertula", the Uncultivated Microbial Symbiont of the Marine Bryozoan Bugula Neritina. J. Nat. Prod. 2007, 70, 67-74.

(54) Walther, E.; Boldt, S.; Kage, H.; Lauterbach, T.; Martin, K.; Roth, M.; Hertweck, C.; Sauerbrei, A.; Schmidtke, M.; Nett, M. Zincophorin - Biosynthesis in Streptomyces Griseus and Antibiotic Properties. GMS Infect. Dis. 2016, 4, Doc08.

(55) Wagner, D. T.; Zhang, Z.; Meoded, R. A.; Cepeda, A. J.; Piel, J.; Keatinge-Clay, A. T. Structural and Functional Studies of a Pyran Synthase Domain from a Trans-Acyltransferase Assembly Line. ACS Chem. Biol. 2018, 13, 975-983.

(56) Li, Y.; Dodge, G. J.; Fiers, W. D.; Fecik, R. A.; Smith, J. L.; Aldrich, C. C. Functional Characterization of a Dehydratase Domain from the Pikromycin Polyketide Synthase. J. Am. Chem. Soc. 2015, 137, 7003-7006.

(57) Fiers, W. D.; Dodge, G. J.; Sherman, D. H.; Smith, J. L.; Aldrich, C. C. Vinylogous Dehydration by a Polyketide Dehydratase Domain in Curacin Biosynthesis. J. Am. Chem. Soc. 2016, 138, 16024-16036.

(58) Bali, S.; O’Hare, H. M.; Weissman, K. J. Broad Substrate Specificity of Ketoreductases Derived from Modular Polyketide Synthases. ChemBioChem 2006, 7, 478-484.

(59) Bali, S.; Weissman, K. J. Ketoreduction in Mycolactone Biosynthesis: Insight into Substrate Specificity and Stereocontrol from Studies of Discrete Ketoreductase Domains in Vitro. ChemBioChem 2006, 7, 1935-1942.

(60) Huerta, F. F.; Minidis, A. B. E.; Bäckvall, J.-E. Racemisation in Asymmetric Synthesis. Dynamic Kinetic Resolution and Related Processes in Enzyme and Metal Catalysis. Chem. Soc. Rev. 2001, 30, $321-331$

(61) Turner, N. J. Deracemisation Methods. Curr. Opin. Chem. Biol. 2010, 14, 115-121.

(62) Voss, C. V.; Gruber, C. C.; Faber, K.; Knaus, T.; Macheroux, P.; Kroutil, W. Orchestration of Concurrent Oxidation and Reduction Cycles for Stereoinversion and Deracemisation of Sec-Alcohols. J. Am. Chem. Soc. 2008, 130, 13969-13972.

(63) Voss, C. V.; Gruber, C. C.; Kroutil, W. Deracemization of Secondary Alcohols through a Concurrent Tandem Biocatalytic Oxidation and Reduction. Angew. Chem., Int. Ed. 2008, 47, 741-745.

(64) Haak, R. M.; Berthiol, F.; Jerphagnon, T.; Gayet, A. J. A.; Tarabiono, C.; Postema, C. P.; Ritleng, V.; Pfeffer, M.; Janssen, D. B.; Minnaard, A. J.; Feringa, B. L.; de Vries, J. Dynamic Kinetic Resolution of Racemic $\beta$-Haloalcohols: Direct Access to Enantioenriched Epoxides. J. Am. Chem. Soc. 2008, 130, 13508-13509.

(65) Mutti, F. G.; Orthaber, A.; Schrittwieser, J. H.; Vries, J. G.; Pietschnig, R.; Kroutil, W. Simultaneous Iridium Catalysed Oxidation and Enzymatic Reduction Employing Orthogonal Reagents. Chem. Commun. 2010, 46, 8046-8048.

(66) Pellissier, H. Recent Developments in Dynamic Kinetic Resolution. Tetrahedron 2011, 67, 3769-3802.

(67) Hoyos, P.; Pace, V.; Alcántara, A. R. Dynamic Kinetic Resolution via Hydrolase-Metal Combo Catalysis in Stereoselective Synthesis of Bioactive Compounds. Adv. Synth. Catal. 2012, 354, 2585-2611.

(68) Martín-Matute, B.; Edin, M.; Bogár, K.; Bäckvall, J.-E. Highly Compatible Metal and Enzyme Catalysts for Efficient Dynamic Kinetic Resolution of Alcohols at Ambient Temperature. Angew. Chem., Int. Ed. 2004, 43, 6535-6539.

(69) Rulli, G.; Heidlindemann, M.; Berkessel, A.; Hummel, W.; Gröger, H. Towards Catalyst Compartimentation in Combined Chemo- and Biocatalytic Processes: Immobilization of Alcohol Dehydrogenases for the Diastereoselective Reduction of a $\beta$-Hydroxy 
Ketone Obtained from an Organocatalytic Aldol Reaction. J. Biotechnol. 2013, 168, 271-276.

(70) Heidlindemann, M.; Rulli, G.; Berkessel, A.; Hummel, W.; Gröger, H. Combination of Asymmetric Organo- and Biocatalytic Reactions in Organic Media Using Immobilized Catalysts in Different Compartments. ACS Catal. 2014, 4, 1099-1103.

(71) Aragão Craveiro, A.; da Costa Prado, A.; Gottlieb, O. R.; Welerson de Albuquerque, P. C. Diarylheptanoids of Centrolobium Species. Phytochemistry 1970, 9, 1869-1875.

(72) de C Alcântara, A. F.; Souza, M. R.; Piló-Veloso, D. Constituents of Brosimum Potabile. Fitoterapia 2000, 71, 613-615.

(73) Boulard, L.; BouzBouz, S.; Cossy, J.; Franck, X.; Figadère, B. Two Successive One-Pot Reactions Leading to the Expeditious Synthesis of (-)-Centrolobine. Tetrahedron Lett. 2004, 45, 66036605.

(74) Chandrasekhar, S.; Prakash, S. J.; Shyamsunder, T. Asymmetric Synthesis of the Pyran Antibiotic (-)-Centrolobine. Tetrahedron Lett. 2005, 46, 6651-6653.

(75) Colobert, F.; Mazery, R. D.; Solladié, G.; Carreño, M. C. First Enantioselective Total Synthesis of (-)-Centrolobine. Org. Lett. 2002, 4, 1723-1725.

(76) Clarke, P. A.; Martin, W. H. C. Exploiting the Maitland-Japp Reaction: A Synthesis of ( \pm -Centrolobine. Tetrahedron 2005, 61, 5433-5438.

(77) Goldsmith, M.; Tawfik, D. S. Enzyme Engineering: Reaching the Maximal Catalytic Efficiency Peak. Curr. Opin. Struct. Biol. 2017, $47,140-150$. 